\title{
Deciphering the Regulation of the Mannitol Operon Paves the Way for Efficient
} Production of Mannitol in Lactococcus lactis

\author{
Xiao, Hang; Bang-Berthelsen, Claus Heiner; Jensen, Peter Ruhdal; Solem, Christian
}

Published in:

Applied and Environmental Microbiology

Link to article, DOI:

10.1128/AEM.00779-21

Publication date:

2021

Document Version

Peer reviewed version

Link back to DTU Orbit

Citation (APA):

Xiao, H., Bang-Berthelsen, C. H., Jensen, P. R., \& Solem, C. (2021). Deciphering the Regulation of the Mannitol Operon Paves the Way for Efficient Production of Mannitol in Lactococcus lactis. Applied and Environmental Microbiology, 87(16). https://doi.org/10.1128/AEM.00779-21

\section{General rights}

Copyright and moral rights for the publications made accessible in the public portal are retained by the authors and/or other copyright owners and it is a condition of accessing publications that users recognise and abide by the legal requirements associated with these rights.

- Users may download and print one copy of any publication from the public portal for the purpose of private study or research.

- You may not further distribute the material or use it for any profit-making activity or commercial gain

- You may freely distribute the URL identifying the publication in the public portal 
(1) AEM Accepted Manuscript Posted Online 9 June 2021

Appl Environ Microbiol doi:10.1128/AEM.00779-21

Copyright $\odot 2021$ American Society for Microbiology. All Rights Reserved.

1

1

2

3

4

5

6 Abstract

7 Lactococcus lactis has great potential for high-yield production of mannitol, which has not

8 yet been fully realized. In this study, we characterize how the mannitol genes in L. lactis are

9 organized and regulated, and use this information to establish efficient mannitol production.

10

11

12
Deciphering the regulation of the mannitol operon paves the way for efficient production of mannitol in L. lactis

Hang Xiao, Claus Heiner Bang-Berthelsen, Peter Ruhdal Jensen* and Christian Solem*

National Food Institute, Technical University of Denmark, DK-2800 Kongens Lyngby,

Denmark
Although the organization of the mannitol genes in L. lactis was similar to that in other Gram-positives, $m t l F$ and $m t l D$, encoding the Enzyme IIA component (EIIA ${ }^{\mathrm{mtl}}$ ) of the mannitol phosphotransferase system (PTS), and the mannitol-1-phosphate dehydrogenase, respectively, were separated by a transcriptional terminator, and the mannitol genes were found to be organized in two transcriptional units: an operon comprising mtlA, encoding the Enzyme IIBC component (EIIBC ${ }^{\mathrm{mtl}}$ ) of the mannitol PTS, $m t l R$, encoding a transcriptional activator, and $m t l F$, and a separately expressed $m t l D$. The promoters driving expression of the two transcriptional units were somewhat similar, and both contained predicted catabolite responsive elements ( $\mathrm{cre}$ ). Presence of carbon catabolite repression was demonstrated, and was shown to be relieved in stationary phase cells. The transcriptional activator MtlR $(m t l R)$, in some Gram-positives, is repressed by phosphorylation by EIIA ${ }^{\mathrm{mtl}}$, and when we knockedout $m t l F$ we indeed observed enhanced expression from the two promotors, which indicated that this mechanism was in place. Finally, by overexpressing the $m t l D$ gene and using stationary phase cells as biocatalysts, we attained $10.1 \mathrm{~g} / \mathrm{L}$ mannitol with a $55 \%$ yield, which 
is the highest titer ever reported for L. lactis. Summing up, the results of our study should be useful for improving the mannitol producing capacity of this important industrial organism.

Importance

Lactococcus lactis is the most studied species of the Lactic Acid Bacteria, and it is widely used in various food fermentations. To date, there have been several attempts to persuade $L$. lactis into producing mannitol, a sugar alcohol with important therapeutic and food applications. Until now, to achieve mannitol production in L. lactis, with significant titer and yield, it has been necessary to introduce and express foreign genes, which precludes the use of such strains in foods, due to their recombinant status. In this study, we systematically characterize how the mannitol genes in L. lactis are regulated, and demonstrate how this impacts on mannitol production capability. We harness this information and manage to establish efficient mannitol production, without introducing foreign genes.

\section{Key words}

L. lactis, mannitol, $m t l R, m t l D, c c p A$

\section{Introduction:}

Lactococcus lactis (L. lactis) is a lactic acid bacterium (LAB) with a long history of safe use in the dairy and food industry. Due to its safe status, its well-known metabolism, and the many genetic tools that have been developed for it, L. lactis is regarded as an excellent production platform for food ingredients as well as for compounds with sensitive applications, such as therapeutics (1). Mannitol, a sugar alcohol, is an example of a compound that can be produced in many LAB, including L. lactis $(2,3)$. Different from heterologous fermentative LAB, which produce mannitol rely on reducing fructose, L. lactis and other homologous fermentative $\mathrm{LAB}(4,5,6)$ produce mannitol rely on reducing fructose-6-phosphate (F6P), which is a common intermediate in the glycolytic process. This means L. lactis could 
theoretically produce mannitol from all carbon sources that are metabolized to F6P, which would further broaden its application as a mannitol producer. Although mannitol production in L. lactis has been studied to some extent during the last two decades, still many aspects of mannitol production are unclear $(7,8)$.

Mannitol biosynthesis in L. lactis, and other homofermentative lactic acid bacteria, starts with the glycolysis intermediate (F6P). The mannitol 1-phosphate dehydrogenase (M1PDH) is responsible for reducing F6P into mannitol 1-phosphate (M1P), which subsequently is dephosphorylated to mannitol by an uncharacterized phosphatase. The normal function of M1PDH is not to facilitate mannitol production, but rather growth on mannitol, and its encoding gene $m t l D$ is located together with genes coding for the mannitol specific PEP (phosphoenolpyruvate)-PTS components $(m t l A \& m t l F)$ and the gene for the regulator $(m t l R)$. Although L. lactis has the metabolic capacity for making mannitol, the relevant genes are under tight control and appear not to be expressed on glucose.

In most Gram-positive bacteria, the transcription activator MtlR controls the expression of the genes encoding M1PDH, and the D-mannitol-specific PTS components (9). MtlR from different Gram-positive bacteria have been studied and they all share domains that have different regulatory functions depending on organism. In general, MtlR contains an Nterminal helix-turn-helix motif followed by an Mga-like domain, two PTS regulation domains (PRDs), an EIIB ${ }^{\mathrm{Gat}}$-like, and an EIIA ${ }^{\mathrm{Mtl}}$-like domain (Fig. 1). There are exceptions, however, and, for instance, MtIR in Lactobacillus casei lacks the PRD1 and EIIB ${ }^{\mathrm{Gat}}$-like domains. There are several regulatory sites in MtlR that can be phosphorylated by Enzyme IIs (EIIs) and HPr, which can activate or inhibit the activity of MtlR. In the presence of an efficiently metabolized PTS substrate, e.g. glucose, HPr is barely phosphorylated (10), and this prevents activation of MtlR. Another regulatory mechanism, which turns off MtlR when mannitol is absent, is the mannitol specific PTS component EIIA, which can phosphorylate the EIIB ${ }^{\text {Gat }}$ 
like domain of MtlR, leading to inactivation of MtlR (11). In addition to these forms of regulation the classical carbon catabolite repression (CCR) is also involved in the regulation of the mannitol operon (12) and together these three regulatory mechanisms probably explain why the mannitol genes normally are repressed during growth on other sugars, and why mannitol is not produced.

There have been many efforts to achieve mannitol production in L. lactis. Gaspar et al. (13), reported that an L. lactis strain deficient in lactate dehydrogenase (ldh) formed small amounts of mannitol $(0.7 \mathrm{mM})$ when growing on glucose, however, after glucose depletion mannitol was metabolized. The same researchers also found that non-growing cells resuspended in buffer, were able to convert $33 \%$ of the glucose metabolized into mannitol. Wisselink et al. demonstrated that the M1PDH had an impact on mannitol production, but the effect was only seen clearly in a lactate dehydrogenase deficient strain, even under nongrowing conditions (14). By heterologous overexpression of the mannitol-1-phosphatase from Eimeria tenella and a M1PDH from Lactobacillus plantarum in a LDH deficient strain, efficient mannitol production $(9.0 \mathrm{~g} / \mathrm{L}, 50 \%$ yield $)$ was accomplished in growing L. lactis cells for the first time (15). All reported cases of mannitol production using growing cells, involve introduction of foreign genes in L. lactis, in combination with additional modifications to metabolism. Since such strains are genetically engineered, their use in food fermentations is precluded. To overcome this problem we recently attempted a different strategy, namely adaptive evolution on mannitol, where the aim was to debottleneck mannitol catabolism, in the hope that this could result in more efficient production of mannitol from glucose. It was indeed possible to enhance growth on mannitol, and the mutants obtained contained a mutation upstream mtlA, which greatly enhanced expression of the mannitol genes. By further eliminating competing NADH consuming pathways, it was possible to 
achieve mannitol production from glucose with high yield $(60 \%)$ and fairly high titer $(6.1 \mathrm{~g} / \mathrm{L})$ using a two-stage fermentation setup (16).

In this study, we further characterize the effects of those mutations and study the roles that MtlR and CcpA have in regulating mannitol production from glucose. Based on the knowledge accumulated we manage to establish efficient mannitol production using stationary phase cells, and achieve the highest titer and yield ever reported, when relying solely on native metabolism.

\section{Results}

\section{Organization of the mannitol genes in L. lactis}

As mentioned above, we recently reported that adaptive laboratory evolution combined with systematic elimination of $\mathrm{NADH}$ consuming reactions can lead to efficient mannitol production, by L. lactis, from glucose. The strains obtained after adaptive evolution contained two different mutations, C-39T and G-46T, both in the mtlA promoter region, which we found were critical for mannitol production (16).

To better understand the effect of these mutations, we first examined the genetic arrangement of the mannitol genes in L. lactis and compared our findings to those previously reported for other Gram-positive bacteria. As shown in Fig. 2a, the mannitol genes appear to be organized in a similar manner in all the bacteria investigated, except for $B$. subtilis, where the gene encoding the regulator MtlR is distantly located from the other mannitol genes. Nevertheless, we observed two distinct features for the L. lactis genes. The first one was that the spaces between $m t l R \& m t l F$, and between $m t l F \& m t l D$ appear longer in L. lactis, than in other organisms. Another difference is that there is a predicted rho-independent terminator between $m t l F$ and $m t l D$, suggesting that $m t l D$ is transcribed from its own promoter. Interestingly, the gene just after $m t l D$, encoding a characterized $6 \mathrm{~S}$ (17) RNA may also be 
121

affected by the promoters upstream of $m t l D$, since no transcriptional terminator can be identified between the 6S RNA gene and $m t l D$.

The promoter regions upstream $m t l A$ and $m t l D$ in L. lactis were also analyzed, and interestingly, we found that they share more than $47 \%$ identity. Both regions have putative MtlR binding sequences and cre sites that are well-conserved. Furthermore, as shown in Fig. $2 \mathrm{~b}$, the two mutations, C-39T and G-46T were both found to locate in the predicted putative cre site, where C-39T also overlapped with the putative -10 element region, suggesting that the mutations affect the expression of mannitol operon.

\section{Adaptive laboratory evolution on mannitol lead to mutations which enhance the mtlA promoter}

To assess the effect of these mutations on expression from the mtlA promoter, transcriptional fusions between the mtlA promoter and the reporter gene gusA (encoding $\beta$ glucuronidase) were constructed based on the reporter vector pTD6. As shown in Table 2, on glucose, a low promoter activity, no more than 0.1 MU (Miller Units), was observed for the wild-type promoter. In contrast, the promoters containing the C-39T or G-46T mutations were significantly stronger, in particular the C-39T mutation, which was 40 fold stronger than the promoter containing the G-46T mutation. The $\beta$-glucuronidase activity measured, depended on host, e.g. the activity of the C-39T promoter in the mannitol adapted strain MG1363M, having the C-39T mutation in its genome, was $52.16 \mathrm{MU}$, whereas the activity in the wild-type strain MG1363 only reached 15.6 MU. As MtlR is expressed together with mtlA and the mannitol PTS genes (one transcriptional unit), the C-39T mutation in MG1363M would result in higher expression of MtlR, which could in turn explain the higher activity of the mtlA promoter in the MG1363M background. On mannitol, as expected, we 
found a higher activity, and whereas the wild-type promoter reached $449 \mathrm{MU}$, the C-39T promoter activity resulted in more than $4000 \mathrm{MU}$.

Based on this information, the promoter mutations in the adapted strains appear to enhance the expression of $m t l A, m t l R$ and $m t l F$ and possibly $m t l D$. We previously found that deleting $m t l F$ in L. lactis had a beneficial effect on mannitol production (16), while others have found that strains lacking either $m t l A$ or $m t l F$ perform similarly in terms of mannitol production (13).

\section{The promoters upstream $m t l A\left(\mathbf{P}_{m t l A}\right)$ and $\mathbf{m t l D}\left(\mathbf{P}_{\text {mtID }}\right)$ are both activated by MtIR and}

\section{drive expression of separate transcriptional units}

To investigate the promoter upstream $m t l D$, we inserted it into the promoter probe vector pTD6, and introduced the plasmid in AceR, the $m t l R$ depleted strain derived from Ace001M (Ace001 adapted on mannitol, containing the C-39T mutation in mtlA promoter). We also introduced the previously constructed $m t l A$ promoter-gusA fusion in the same strain. As shown in Fig. 3, on glucose, in the AceR background, we found that both promoters were less active than in the parental strain Ace001M. In Ace001M the mannitol operon is expressed to a higher level, leading to more of the activator MtlR. These findings clearly demonstrated that MtlR was an activator of the promoter upstream $m t l D$. Interestingly, the promoter activities were much higher for cells in stationary phase than in exponential phase and for both promoters, a large induction was observed on mannitol.

To investigate if the expression of $m t l D$ could be affected by the promoter upstream $m t l A$ $\left(\mathrm{P}_{\mathrm{mtl}}\right)$, we fused the mutated mtlA promoter $(\mathrm{C}-39 \mathrm{~T})$ with the $m t l D$ promoter, including the transcriptional terminator upstream $m t l D$, and introduced the construct into the reporter vector pTD6. In the Ace001M background, a low expression level was detected, only $1.82 \pm 0.13 \mathrm{MU}$, which is similar to that found for $m t l D$ promoter alone. This demonstrates that $m t l D$ is not part of the $m t l A-m t l R-m t l F$ operon. 


\section{Deleting cсpA increases expression of the mannitol genes}

The mannitol genes are subject to carbon catabolite repression, and this might have a negative effect on mannitol production from glucose. To investigate this, we deleted $\operatorname{ccp} A$ in Ace001, thereby generating Ace001C. As shown in Fig. 4, by deleting $c c p A$, the $m t l A$ and the $m t l D$ promoters were both derepressed, and activities of $588 \mathrm{MU}$ and $405 \mathrm{MU}$, respectively were measured in the exponential phase (Table 3). Furthermore, we found that the wild type $m t l A$ promoter and $m t l A$ promoter from the mannitol adapted strains had similar activities in the $\triangle c c p A$ background, demonstrating that the mutation had affected the functionality of the cre site (Fig. 4). Moreover, two to three fold higher promoter activity were detected in the stationary phase.

\section{MtlR is needed for high-level expression of the mannitol genes, and its level can be} enhanced by deleting $m t l F$

For investigating the extent to which MtlR affects expression of mannitol genes, we deleted cсpA in AceR, which lacks mtlR, thus generating AceCR. As shown in Fig. 5, compared with AceR (Fig. 3), deleting ccpA (AceCR) only had a small positive effect on the $m t l A$ promoter, and the $m t l D$ promoter had no activity. These results clearly demonstrate that the mannitol genes in L. lactis are tightly regulated by MtlR. To investigate whether eliminating CcpA could be beneficial for mannitol production, we deleted $c c p A$ in strain AceF, which harbors the C-39T promoter mutation and in which $m t l F$ has been deleted; the outcome was strain AceCF. As observed earlier, without $m t l F$, the activity of $m t l A$ and $m t l D$ promoters were dramatically increased to 3787 MU and 1955 MU, respectively, in the exponential phase, and increased to $6055 \mathrm{MU}$ and $3664 \mathrm{MU}$ in the stationary phase (Fig. 5 and Table 3).

\section{Inactivating CcpA enables mannitol production in non-mannitol adapted strains}


To quantify the effect of MtlR on the expression level of the mannitol genes, on mannitol catabolism and on mannitol production from glucose, we overexpressed $m t l R$ from a promoter of intermediate strength in the non-mannitol producer Ace001 (MG1363 4 ldh $\triangle l d h B \Delta l d h X \triangle p t a \triangle a d h E \triangle b u t B A)$. As shown in Table 4, this enabled mannitol production from glucose. Deleting $m t l R$ in the mannitol producer Ace001M, abolished both growth on mannitol and mannitol production from glucose, which indicates that MtlR has a key role in both mannitol catabolism and production. AceCF and AceF were both able to produce mannitol, but unable to grow on mannitol, where the latter was expected, since $m t l F$ encodes a critical mannitol PTS component.

\section{MtIR has no influence on the mannitol-1-phosphatase activity}

In L. lactis, two activities are needed for mannitol production to occur (Fig. 6); the first is mannitol-1-phosphate dehydrogenase $(m t l D)$, and the second is a phosphatase activity that can convert mannitol-1-phosphate into mannitol, where the latter has not been identified. The impact that MtlR has on the expression of the mannitol genes is clear, however, is it possible that MtlR could serve as an M1Pase or be able to increase the M1Pase activity in cells, as MtlR contains several phosphoryl transfer domains. For testing this, we measured the M1pase activity in Ace001-mtlR and AceR. We finally detected similar M1Pase activity in Ace001mtlR AceR and Ace001 (0.48, 0.49 and 0.49 respectively). Based on these results, we concluded that MtlR can not be responsible for the M1Pase activity in L. lactis.

\section{Mannitol production using two-step fermentation from different strains}

As deletion of $c c p A$ leads to increased expression of the mannitol genes, we decided to test if this could also be beneficial for mannitol production. We therefore tested AceCF and AceF in the two-step fermentation setup used previously (16). As shown in Table 5, AceCF, the strain lacking cсpA had similar performance with its parental strain AceF, both in terms of 
titer and yield for mannitol. One possible explanation is that glucose metabolism is hampered to such an extent that the beneficial effect coming from overexpressing the mannitol genes is lost. For AceR-mtlD, a strain that overexpresses $m t l D$ and lacks MtlR, surprisingly a titer of $10.1 \mathrm{~g} / \mathrm{L}$ with a $55 \%$ yield was attained, which is the highest ever reported for L. lactis. This clearly demonstrated the key role of the mannitol 1-phosphate dehydrogenase in mannitol production. Furthermore, we also tested if the strains AceCF and AceR-mtlD were able to produce mannitol under semi-anaerobic growth conditions, where slow diffusion of oxygen into the culture medium was allowed (no active aeration), and indeed, slow growth and mannitol production was observed.

\section{Discussion}

In L. lactis, mtlD encodes M1PDH, which is a key enzyme in mannitol metabolism. In this study, we found that MtlR is essential for maintaining the expression of $m t l D$, thus allowing for mannitol production in non-growing or slowly growing cells.

\section{The phosphatase responsible for dephosphorylating mannitol-1-phosphate remains to be found}

Although several studies have shown that L. lactis is able to convert M1P into mannitol, the responsible enzyme has not been identified $(13,18)$. It has been suggested that a mannitol specific PTS component could be involved in this, e.g. the EIIBC ${ }^{\mathrm{mtl}}$, as it is able to transfer phosphoryl group between M1P and mannitol. However, Ramos and coworkers showed that neither EIIBC ${ }^{\mathrm{mtl}}$ nor EIIA ${ }^{\mathrm{mtl}}$ are responsible for the M1Pase activity in L. lactis (13). In this study, we clarified that the other phosphoryl transferring protein MtlR also is not responsible for the M1Pase activity. Inducer expulsion, which is a unique mechanism only found in few Gram-positive bacteria, including L. lactis, could be the explanation. Inducer expulsion is the process where intracellular sugar phosphates are dephosphorylated and exported when 
preferred sugars, like glucose are present $(19,20,21,22,23)$. In our case, when cells are incubated with glucose, M1P accumulates, is dephosphorylated to mannitol, and transported out of the cell. Interestingly, in L. lactis a $10 \mathrm{kDa}$ sugar-phosphatase having a broad substrate specificity was characterized and found to be responsible for inducer expulsion $(24,25)$. If this enzyme is the M1Pase, we probably could overcome the bottleneck in mannitol production by enhancing its expression, thus avoiding heterologous gene expression (15).

\section{CCR is relieved when cells reach stationary phase}

CcpA is a global transcriptional regulator, which is responsible for CCR in Gram-positive bacteria. It functions as a complex with P-Ser-Hpr, and exerts CCR by binding to the cre site upstream of various genes. In this study, we validated that CcpA serves as a strong repressor, preventing expression of the mannitol genes when glucose is present. We characterized several mutants adapted to growth on mannitol, and in one of these a mutation was found in the mtlA promoter region (C-39T mutant). In the C-39T mutant, the mtlA promoter had become more active, both on glucose but especially on mannitol. However, after inactivating the $\operatorname{ccp} A$ gene, the $\mathrm{C}-39 \mathrm{~T}$ promoter displayed a similar activity as the wild type promoter. This indicates that the C-39T mutation had an influence on the functionality of the cre site in this promoter. In stationary phase cells, the mannitol genes appeared to be upregulated around 50-fold as compared to the exponential phase. This provided an explanation for why the two-step fermentation approach used previously resulted in a high mannitol production in the presence of glucose, which promotes catabolite repression (16). This de-repression could be due to a decreased level of P-Ser-Hpr in the cells, and it has been reported that Hpr mainly exists in the dephosphorylated form in stationary phase cells (26). We speculate that a decreased level of CcpA could also contribute to the observed behavior, however, to clarify this further studies are needed, eg., where the transcriptome of stationary phase cells is scrutinized. 
Expression of a 6S RNA gene located downstream $m t l D$, is influenced by the $m t l D$ promoter

van der Meulen et al. characterized a 6S RNA gene located 105 bp downstream of mtlD (17). Interestingly, it was shown that the expression of this gene was upregulated by 3 fold after deletion of the cсpA gene, and by growing the cells in galactose or cellobiose, repression could also be relieved. Although there is a predicted promoter and a cre site upstream of this gene, there is no predicted transcriptional terminator between this gene and $m t l D$, suggesting that increased expression of the $m t l D$ gene could positively affect the expression of this $6 \mathrm{~S}$ RNA gene. In addition, it was also shown that several noncoding RNAs and protein coding genes are regulated by this 6S RNA, and it was deduced to be active when CCR is relieved during stationary phase and/or growth on alternative carbon sources (17).

\section{L. lactis MtIR is different from homologs in other firmicutes}

In this study, we substantiated that MtlR is indeed a transcriptional activator, which plays an important role for the expression of mannitol genes, and we characterized a mutant $m t l A$ promoter found in a mannitol adapted strain, which grows fast on mannitol. The mutated mtlA promoter retained an activity of around $40 \mathrm{MU}$ in the absence of mannitol, which appears to be due to less carbon catabolite repression, as the mutation is located in the predicted cre site. In B. subtilis, MtlR binds to an incomplete inverse repeat sequence upstream of the mtlA promoter (11). In L. lactis, a similar inverse repeat sequence can also be found upstream of both $m t l A$ and $m t l D$ (Fig. 2b). Interestingly, these inverse repeat sequences are likely to form rho-independent terminators, as they contain a GC rich harpin following by several Ts. From a protein alignment between studied MtlRs from other Gram-positive bacteria, we found that L. lactis MtlR is much more similar to the S. mutans MtlR (supplementary table 1). However, in one study it was claimed that MtlR in S. mutans was 
not needed for expression of the mannitol genes, and that this was due to a long insertion in the $m t l R$ gene (27). Despite of this, in most other studies of MtlR in firmicutes, it has been shown that MtlR indeed acts as transcriptional activator for the mannitol operon $(28,29,30$, 31, 32, 33). MtlR generally consist of two parts. The first part is the helix-turn-helix Mga domain, which was first characterized in the S. pyogens virulence gene regulator Mga, where it is responsible for binding to its DNA target. The second part consists of PRD domains and EIIA $^{\text {Mtl }}$-like domains (as well as EIIB ${ }^{\text {Gat }}$-like domains in some firmicutes), which regulate the activity of MtlR through their phosphorylation states. In general, it has been found that the PRD1 domain has little effect on the activity of MtlR, whereas the phosphorylation status of the PRD2 domain greatly affects activity, where phosphorylation activates MtlR. Furthermore, dephosphorylation of the $\mathrm{EIIA}^{\mathrm{Mtl}}$-like and $\mathrm{EIIB}^{\mathrm{Gat}}$-like domains has positive effects on the activity of MtlR. Interestingly, the L. casei MtlR is only regulated by its EIIA $^{\mathrm{Mtl}}$-like domain (9). By sequence alignment with other characterized MtlR and domain prediction, L.lactis MtlR appears structurally similar to L. casei MtlR, which does not have PRD1 and EIIB ${ }^{\text {Gat }}$-like domains (supplementary Figure 1, 2). However, the histidine in the PRD2 domain is conserved in L. lactis MtlR. Moreover, in B. subtilis, it was shown that regulation of the mannitol operon mainly takes place via phosphorylation of MtlR, and that CcpA mediated carbon catabolite repression does not play a significant role, as expression of mannitol genes was not significantly affected in a ccpA deficient strain (12). Differently, in $L$. lactis, we have shown CcpA is a strong repressor of the mannitol genes, while MtlR seemingly is still active in the presence of glucose. In G. stearothermophilus and L. casei, phosphorylation in the EIIA ${ }^{\mathrm{Mtl}}$-like domain by $\mathrm{P} \sim \mathrm{EIIB}^{\mathrm{Mtl}}$ inhibits the activity of MtlR $(9,34)$.

Several studies have shown that mannitol production can be enhanced by deleting $m t l F$, and it has been concluded that this is due to prevention of re-uptake of the mannitol produced (16, 35, 13). In this study, we detected a four-fold higher MtlR activity in a mtlF (encoding 
314 EIIA ${ }^{\mathrm{Mtl}}$ ) deficient stain, where $\mathrm{EIIB}^{\mathrm{Mtl}}$ always is in an unphosphorylated state because of the 315 absence of its phosphoryl group donor EIIA ${ }^{\mathrm{Mtl}}$. This suggests that the EIIA ${ }^{\mathrm{Mtl}}$-like domain in L. lactis MtIR might be functional, and that phosphorylation of the EIIA ${ }^{\mathrm{Mtl}}$-like domain could negatively affect the activity of $L$. lactis MtlR (Fig. 7). If so, deleting the $m t l F$ gene not only inhibits uptake of mannitol from the medium, but also to enhance the M1PDH activity by activating MtlR, thus stimulating mannitol production in L. lactis.

\section{Deleting CcpA is not a good strategy for enhancing mannitol production in L. lactis}

In this study, we have shown that there is an important role for MtlR in mannitol production. However, the sole role of MtlR appears to be in activating expression of the $m t l D$ gene. In an attempt to improve mannitol production from glucose, we deleted the $c c p A$ gene, which resulted in high constitutive expression of the $m t l D$ promoter. Unfortunately, we found that both titer and yield did not change when deleting cсpA. In L. lactis, when $c c p A$ is deleted, glycolysis is hampered $(36,37,38)$. Since PTS uptake of glucose requires phosphoenolpyruvate (PEP), a glycolytic intermediate, this will have a direct effect on mannitol production, which is what we indeed observed. It appears that a more promising strategy for relieving carbon catabolite repression would be to inactivate the CcpA binding site upstream of $m t l A$ and $m t l D$, without reducing the activities of these promoters, which perhaps could be accomplished by classical mutagenesis followed by screening.

In our previous study, to achieve a high mannitol titer, we relied on a two-stage production setup, where we first accumulated biomass and then subsequently used this biomass for producing mannitol (16). In this study, we found out that the success of this approach was mainly due to the relief of CCR and high expression of $m t l D$ in stationary phase cells. To bypass the CCR, we tested whether overexpression of $m t l D$, the mannitol-1-phosphat dehydrogenase, would enable anaerobic growth and mannitol production, however, this was 
not the case. It appears that the mannitol-1-phosphatase is a bottleneck in mannitol production, and irrespective of a high mannitol-1-phosphate dehydrogenase activity, anaerobic $\mathrm{NAD}^{+}$regeneration will remain hampered as long as the phosphatase activity is limiting, and the only way to achieve a high mannitol productivity is using dense cell suspensions. We also speculated whether the cells could be starving for ATP, since mannitol production is ATP demanding. To ameliorate the latter, we supplemented the cell suspensions with arginine, that can be metabolized via the arginine deiminase pathway leading to ATP formation (39). This indeed had a beneficial effect, however, only a small one. Boosting the inherent mannitol-1-phosphatase activity, e.g. by mutagenesis, thus appears to be a first priority if a successful mannitol production in L. lactis is to be established.

\section{Materials and methods}

\section{Bacterial Strains and Growth Conditions}

Lactococcus lactis subsp. cremoris MG1363 and its derivatives were used in this study (described in Table 1). L. lactis strains were cultured in M17 broth supplemented with $1 \%$ glucose (Sigma Aldrich) at $30^{\circ} \mathrm{C}$, with shaking at $240 \mathrm{rpm}$. When needed $5 \mu \mathrm{g} / \mathrm{ml}$ tetracycline was used for selection, and $2 \mu \mathrm{g} / \mathrm{ml}$ in physiological growth experiments. The optical density of cell suspensions was measured using a spectrophotometer (Shimadzu) at $600 \mathrm{~nm}$. Resting cells preparation and two-step fermentation were performed as described previously (16).

\section{Methods and tools for bioinformatics analysis}

The information about mannitol genes in different bacteria was obtained from NCBI with accession number: NC_009004 (L. lactis, MG1363), NC_000964 (Bacillus subtilis, str. 168), U18943 (Geobacillus stearothermophilus, ATCC 7954), U53868 (Clostridium acetobutylicum, ATCC 824) and AE014133 (Streptococcus mutans, UA159). The bioinformatics analysis was performed using different webtools: BDGP (40) and SAPPHIRE 
https://sapphire.biw.kuleuven.be/ for promoter prediction; De Novo DNA (https://www.denovodna.com) for predicting ribosome binding site; Arnold (http://rssf.i2bc.paris-saclay.fr/toolbox/arnold) for predicting transcriptional terminators.

\section{Molecular techniques}

Electrocompetent cells were prepared using the protocol of Holo \& Nes (41), using GM17 medium containing either $0.5 \%$ (for strains AceCF \& AceCR) or $2 \%$ glycine and $0.25 \mathrm{M}$ sucrose. The medium was filtered. Electroporation was performed as as previously described by Holo and Nes using MicroPulser Electroporator (Bio-Rad) (41). Phusion DNA Polymerase (Thermofisher Scientific) was used for PCR amplifications. Zyppy plasmid 371 miniprep kit (Zymo Research) was used for extracting the plasmids from L. lactis after a pretreatment with $20 \mathrm{mg} / \mathrm{mL}$ lysozyme (Fluka, $7,000 \mathrm{U} / \mathrm{mg}$ ) at $37^{\circ} \mathrm{C}$ for 2 hours. FastDigest restriction enzymes SalI, SacI and BamHI were obtained from Thermofisher Scientific.

To delete $m t l R$ in the strain Ace001M, $\mathrm{pG}^{+}$host8, a plasmid with a thermosensitive replicon was used. Two synthetic DNA fragments, representing the upstream and 377 downstream regions of $m t l R$, and able to overlap were ordered from Integrated DNA 378 Technologies: a 985 bp fragment upstream of $m t l R$ into which a Sall restriction site had been introduced in the $m t l R$ distal end, and a $993 \mathrm{bp}$ fragment downstream of $m t l R$, with a BamHI restriction site added in the $m t l R$ distal region. After fusing the two fragments they were inserted into pJET1.2 using CloneJET PCR Cloning Kit by blunt ligation as described in the manual and transformed into E. coli $\mathrm{DH} 10 \mathrm{~B}$. The insert was excised as a Sall/BamHI

383 fragment and introduced into the corresponding sites in $\mathrm{pG}^{+}$host8 using E. coli $\mathrm{MC} 1000$ as 384 host. The resulting plasmid, designated pKmtlR ( $\mathrm{pG}^{+}$host $\left.8 / \mathrm{mtlR}_{\mathrm{up}}-\mathrm{mtlR}_{\mathrm{dwn}}\right)$, was 
subsequently used to delete $m t l R$ in Ace001-M in the same manner as described previously (16). The Ace001-M derivative with deleted $m t l R$ was designated AceR.

\section{Construction of strains with deleted $c c p A$}

The same approach used to delete $m t l R$ was taken for deleting $c c p A$. In this case a $978 \mathrm{bp}$ fragment upstream of ccpA equipped with a SalI restriction site and a $984 \mathrm{bp}$ fragment downstream of $c c p A$ with a BamHI site were used. The subsequent cloning steps were identical to the ones mentioned above. For obtaining the strains with deleted $c c p A$, we relied on the slow growth phenotype of $c c p A$ mutants (36), and for this reasons small colonies were selected and investigated. Deletion of $c c p A$ was done for strains AceF, Ace001 and AceR, and the derivatives were designated $\mathrm{AceCF}$, Ace001C and AceCR respectively.

Overexpression of $m t l D$ and $m t l R$

An expression cassette consisting of a promoter, a ribosome binding sequence and $m t l D$ was generated using a nested PCR approach. First, a fragment containing the entire $m t l D$ gene was amplified using primers 5'-ATTTCGGGAGACACATCTGGC-3' and 5'GTGTTCTCGCTTCGCATCAG-3'. For the second PCR a forward primer containing the $20 \%$ TPI promoter sequence (42) and a ribosome binding sequence was used 5'-

\section{CACGCGTCGACATAGATTAGTTTATTCTTGACACTACAAGCTAAATGTGGTATAA} TCCCATAGATATACTAGGTAAGTAATAAAATATTCGGAGGAATTTTGAAATGAA

AAAAGCAGTACATTTTGGTGCAGGAAAT -3' together with the following reverse primer 5'- ATCCGGAGCTCATATTCTCTGTCTACTTGCTGTCAT -3'. The same approach was used for generating an expression cassette for $m t l R$ : first, a fragment containing the entire $m t l R$ gene was amplified using primers 5'- AAGAAGAATTCACGGCAATA -3' and 5'- ATCGCTGAAACATAATTTGAG -3'. For the second PCR the following primers were used: 5'- 
TCCCATAGATATACTAGGTAAGTAATAAAATATTCGGAGGAATTTTGAAATGTTT

TTAACAAGTCGTGAG -3' \& 5'-

ATCCGCTCGAGCTAATCACCATACTGTTTAACAGC -3'. All primers are ordered from

IDT company. The amplified fragments were digested with restriction enzymes and inserted into pTD6 and the constructs introduced into Ace001 and verified by Sanger sequencing (Macrogen).

\section{Quantifying mannitol production by High-Performance Liquid Chromatography} (HPLC)

For quantifying glucose and mannitol, we used an HPLC equipped with a BioRad Aminex HPX-87H column (BioRad) and a RI-101 detector (Shodex). The mobile phase consisted of 5 $\mathrm{mM} \mathrm{H}_{2} \mathrm{SO}_{4}$ at a flow rate of $0.5 \mathrm{ml} / \mathrm{min}$. The column oven temperature was set to $60^{\circ} \mathrm{C}$. The samples for HPLC analysis were filtered using $0.22 \mu \mathrm{M}$ filters (Labsolute) and loaded to HPLC immediately after sampling at appropriate cells density.

\section{M1Pase activity assay}

For detecting M1Pase activity, exponentially growing culture samples were quenched in wet ice. The cells were washed with ice-cold MES buffer (50 mM MES, $10 \mathrm{mM} \mathrm{MgCl}_{2}$, $\mathrm{pH}=7.0$ ), and resuspended to a density of $\mathrm{OD}_{600}=50.0$. For permeabilizing the cells, $12.5 \mu \mathrm{l}$ $0.1 \%$ SDS and $25 \mu \mathrm{CHCl}_{3}$ was added. After vigorous vortex for $10 \mathrm{~s}$ and equilibrium at $30^{\circ} \mathrm{C}$ for $5 \mathrm{~min}$, an appropriate volume of M1P (Sigma Aldrich) was added to a final

429 concentration of $5 \mathrm{mM}$ to initiate the reaction, meanwhile, for negative controls, the same 430 volume of MES buffer was added. After $1 \mathrm{~h}$ incubation at $30^{\circ} \mathrm{C}$, cells were centrifuged and 431 the supernatant was filtered with $0.22 \mu \mathrm{M}$ filters (Labsolute). Finally, the filtered supernatant 
was immediately analyzed by HPLC to determine formation of mannitol. M1Pase activity was descried as the amount of mannitol (mM) formed per hour.

\section{$\beta$-glucuronidase assays}

Strains were cultured in M17 broth supplemented with $1 \%$ of the indicated sugar and 2 $\mu \mathrm{g} / \mathrm{ml}$ tetracycline. Cells were harvested after reaching $\mathrm{OD}_{600}=1.0$ or in the stationary phase $\left(\mathrm{OD}_{600}=4.5\right.$, for strains containing plasmid was $\left.\mathrm{OD}_{600}=4.0\right)$, and quenched on wet ice followed by centrifugation at $4^{\circ} \mathrm{C}$. Harvested cells were washed in ice cold PBS and resuspended in ice-cold $\mathrm{Z}$ buffer $\left(0.06 \mathrm{M} \mathrm{Na}_{2} \mathrm{HPO}_{4}, 0.04 \mathrm{M} \mathrm{NaH}_{2} \mathrm{PO}_{4}, 0.01 \mathrm{M} \mathrm{KCl}, 0.001 \mathrm{M}\right.$ $\mathrm{MgSO}_{4}, 0.05 \mathrm{M} \beta$-mercaptoethanol, $\mathrm{pH}=7$ ). Cells were appropriately diluted and the cell density was measured at $\mathrm{OD}_{600}$. For permeabilization, $12.5 \mu \mathrm{l} 0.1 \% \mathrm{SDS}$ and $25 \mu \mathrm{C} \mathrm{CHCl}_{3}$ were added, and the suspension vortexed vigorously. After equilibrium at $30^{\circ} \mathrm{C}$ for 5 min, $100 \mu \mathrm{l}$ of $4 \mathrm{mg} / \mathrm{ml}$ PNPG was added, and the time needed for developing a yellow color recorded. To stop the reaction $800 \mu \mathrm{Na}_{2} \mathrm{CO}_{3}$ was added. Absorbance at $420 \mathrm{~nm}$ and $550 \mathrm{~nm}$ were measured. Promoter strength was calculated as $1000^{*}\left(\mathrm{OD}_{420^{-}}\right.$ $\left.1.75 * \mathrm{OD}_{550}\right) /\left(\right.$ time $* \mathrm{OD}_{600} * \mathrm{ml}$ sample $)$ and describe as Miller units.

\section{Two-step fermentation procedure for producing mannitol}

For producing mannitol, a two-step fermentation setup was used. First, cells were cultivated in M17 supplemented with $1 \%$ glucose at $30^{\circ} \mathrm{C}, 240 \mathrm{rpm}$ until the cell density reached $\mathrm{OD}_{600}=4.5$ (for strains containing plasmid was $\mathrm{OD}_{600}=4.0$ ). Then cells were shifted to $20 \mathrm{rpm}$ shaking while at the same time, glucose and arginine were added to a final concentration of $2 \%$ and $35 \mathrm{mM}$. Samples were taken at appropriate times and loaded to HPLC immediately, to determine glucose consumption and mannitol production. Mannitol yield was calculated by the mannitol conversion ratio from glucose during stationary period.

\section{Acknowledgements}


456 We thank Jiahuan Tong for drawing figures in this work. This work was supported by the

457 Chinese Scholarship Council (CSC).

\section{References}

1. Neirynck S, Steidler L. 2006. Delivery of therapeutic proteins through Lactococcus lactis. Biotechnol Genet Eng Rev.

2. Song SH, Vieille C. 2009. Recent advances in the biological production of mannitol. Appl Microbiol Biotechnol 84:55-62.

3. Saha BC, Racine FM. 2010. Biotechnological production of mannitol and its applications.

4. De Boeck R, Sarmiento-Rubiano LA, Nadal I, Monedero V, Pérez-Martínez G, Yebra MJ. 2010. Sorbitol production from lactose by engineered Lactobacillus casei deficient in sorbitol transport system and mannitol-1-phosphate dehydrogenase. Appl Microbiol Biotechnol 85:1915-1922.

5. Ladero V, Ramos A, Wiersma A, Goffin P, Schanck A, Kleerebezem M, Hugenholtz J, Smid EJ, Hols P. 2007. High-level production of the low-calorie sugar sorbitol by Lactobacillus plantarum through metabolic engineering. Appl Environ Microbiol $73: 1864-1872$.

6. Biology O. 1976. Short Communications Production of Mannitol 21:551-553.

7. Wisselink HW, Weusthuis RA, Eggink G, Hugenholtz J, Grobben GJ. 2002. Mannitol production by lactic acid bacteria: A review. Int Dairy J 12:151-161.

8. Mitchell H. 2007. Sweeteners and Sugar Alternatives in Food TechnologySweeteners and Sugar Alternatives in Food Technology. 
9. Joyet P, Derkaoui M, Bouraoui H, Deutscher J. 2015. PTS-Mediated Regulation of the Transcription Activator MtlR from Different Species: Surprising Differences despite Strong Sequence Conservation. J Mol Microbiol Biotechnol 25:94-105.

10. Monedero V, Poncet S, Mijakovic I, Fieulaine S, Dossonnet V, Martin-Verstraete I, Nessler S, Deutscher J. 2001. Mutations lowering the phosphatase activity of HPr kinase/phosphatase switch off carbon metabolism. EMBO J 20:3928-3937.

11. Heravi KM, Altenbuchner J. 2014. Regulation of the Bacillus subtilis mannitol utilization genes: Promoter structure and transcriptional activation by the wild-type regulator (MtlR) and its mutants. Microbiol (United Kingdom) 160:91-101.

12. Morabbi Heravi K, Wenzel M, Altenbuchner J. 2011. Regulation of mtl operon promoter of Bacillus subtilis: Requirements of its use in expression vectors. Microb Cell Fact 10:1-19.

13. Ramos A, Gaspar P, Neves AR, Gasson MJ, Santos H, Shearman CA. 2004. Engineering Lactococcus lactis for Production of Mannitol: High Yields from FoodGrade Strains Deficient in Lactate Dehydrogenase and the Mannitol Transport System. Appl Environ Microbiol 70:1466-1474.

14. Wisselink HW, Mars AE, Meer P Van Der, Eggink G, Hugenholtz J. 2004. Metabolic Engineering of Mannitol Production in Lactococcus lactis: Influence of Overexpression of Mannitol 1-Phosphate Dehydrogenase in Different Genetic Backgrounds. Appl Environ Microbiol 70:4286-4292.

15. Wisselink HW, Mars AE, De Vos WM, Hugenholtz J, Moers APHA, Hoefnagel MHN. 2005. Overproduction of heterologous mannitol 1-phosphatase: A key factor for engineering mannitol production by Lactococcus lactis. Appl Environ Microbiol 71:1507-1514. 
16. Xiao H, Wang Q, Bang-Berthelsen CH, Jensen PR, Solem C. 2020. Harnessing Adaptive Evolution to Achieve Superior Mannitol Production by Lactococcus lactis Using Its Native Metabolism. J Agric Food Chem 68:4912-4921.

17. van der Meulen SB, de Jong A, Kok J. 2016. Transcriptome landscape of Lactococcus lactis reveals many novel RNAs including a small regulatory RNA involved in carbon uptake and metabolism. RNA Biol 13:353-366.

18. de Vos WM, Wisselink HW, Hugenholtz J, Moers APHA, Hoefnagel MHN, Mars AE. 2005. Overproduction of Heterologous Mannitol 1-Phosphatase: a Key Factor for Engineering Mannitol Production by Lactococcus lactis. Appl Environ Microbiol $71: 1507-1514$

19. Gasson MJ. 1983. Plasmid complements of Streptococcus lactis NCDO 712 and other lactic streptococci after protoplast-induced curing. J Bacteriol 154:1-9.

20. Thompson J, Saier MH. 1981. Regulation of methyl- $\beta$-D-thiogalactopyranoside-6phosphate accumulation in Streptococcus lactis by exclusion and expulsion mechanisms. J Bacteriol 146:885-894.

21. Monedero V, Kuipers OP, Jamet E, Deutscher J. 2001. Regulatory functions of serine46-phosphorylated HPr in Lactococcus lactis. J Bacteriol 183:3391-3398.

22. Saier MH, Chauvaux S, Cook GM, Deutscher J, Paulsen IT, Reizer J, Ye JJ. 1996. Catabolite repression and inducer control in Gram-positive bacteria. Microbiology 142:217-230.

23. Reizer J, Deutscher J, Sutrina S, Thompson J, Saier MH. 1985. Sugar accumulation in Gram-positive bacteria: exclusion and expulsion mechanisms. Trends Biochem Sci 10:32-35. 
24. Ye JJ, Saier MH. 1995. Purification and characterization of a small membraneassociated sugar phosphate phosphatase that is allosterically activated by $\operatorname{HPr}(\operatorname{Ser}(\mathrm{P}))$ of the phosphotransferase system in Lactococcus lactis. J Biol Chem.

25. Thompson J, Chassy BM. 1983. Intracellular hexose-6-phosphate: phosphohydrolase from Streptococcus lactis: Purification, properties, and function. J Bacteriol 156:70-80.

26. Landmann JJ, Werner S, Hillen W, Stülke J, Görke B. 2012. Carbon source control of the phosphorylation state of the Bacillus subtilis carbon-flux regulator Crh in vivo. FEMS Microbiol Lett 327:47-53.

27. Honeyman AL, Curtiss R. 2000. The mannitol-specific enzyme II (mtIA) gene and the mtIR gene of the PTS of Streptococcus mutans. Microbiology 146:1565-1572.

28. Henstra SA, Tuinhof M, Duurkens RH, Robillard GT. 1999. The Bacillus stearothermophilus Mannitol Regulator, MtlR, of the Phosphotransferase System . J Biol Chem 274:4754-4763.

29. Henstra SA, Duurkens RH, Robillard GT. 2000. Multiple phosphorylation events regulate the activity of the mannitol transcriptional regulator MtlR of the Bacillus stearothermophilus phosphoenolpyruvate-dependent mannitol phosphotransferase system. J Biol Chem 275:7037-7044.

30. Honeyman AL, Curtiss R. 2000. The mannitol-specific enzyme II (mtIA) gene and the mtIR gene of the PTS of Streptococcus mutans. Microbiology 146.

31. Behrens S, Mitchell WJ, Bahl H. 2001. Molecular analysis of the mannitol operon of Clostridium acetobutylicum encoding a phosphotransferase system and a putative PTS-modulated regulator. Microbiology 147:75-86.

32. Bouraoui H, Ventroux M, Noirot-Gros MF, Deutscher J, Joyet P. 2013. Membrane 
sequestration by the EIIB domain of the mannitol permease MtlA activates the Bacillus subtilis mtl operon regulator Mt1R. Mol Microbiol 87:789-801.

33. Joyet P, Derkaoui M, Poncet S, Deutscher J. 2010. Control of Bacillus subtilis mtl operon expression by complex phosphorylation-dependent regulation of the transcriptional activator MtlR. Mol Microbiol 76:1279-1294.

34. Henstra SA, Tuinhof M, Duurkens RH, Robillard GT. $1999 . \quad$ The Bacillus stearothermophilus Mannitol Regulator, MtlR, of the Phosphotransferase System . J Biol Chem 274:4754-4763.

35. Gaspar P, Neves AR, Gasson MJ, Shearman CA, Santos H. 2011. High yields of 2,3butanediol and mannitol in Lactococcus lactis through engineering of NAD + cofactor recycling. Appl Environ Microbiol 77:6826-6835.

36. Gaudu P, Lamberet G, Poncet S, Gruss A. 2003. CcpA regulation of aerobic and respiration growth in Lactococcus lactis. Mol Microbiol 50:183-192.

37. Zomer AL, Buist G, Larsen R, Kok J, Kuipers OP. 2007. Time-resolved determination of the CcpA regulon of Lactococcus lactis subsp. cremons MG1363. J Bacteriol 189:1366-1381.

38. Deutscher J, Küster E, Bergstedt U, Charrier V, Hillen W. 1995. Protein kinase-dependent $\mathrm{HPr} / \mathrm{CcpA}$ interaction links glycolytic activity to carbon catabolite repression in Gram-positive bacteria. Mol Microbiol 15:1049-1053.

39. Crow VL, Thomas TD. 1982. Arginine metabolism in lactic streptococci. J Bacteriol 150:1024-1032.

40. Reese MG. 2001. Application of a time-delay neural network to promoter annotation in the Drosophila melanogaster genome. Comput Chem 26:51-56. 
41. Holo H, Nes IF. 1989. High-frequency transformation, by electroporation, of Lactococcus lactis subsp. cremoris grown with glycine in osmotically stabilized media. Appl Environ Microbiol 55:3119-3123.

42. C. Solem, B. Koebmann PRJ. 2008. Moment-closure approximations for mass-action models. IET Syst Biol 2:64-72.

43. Liu J, Kandasamy V, Würtz A, Jensen PR, Solem C. 2016. Stimulation of acetoin production in metabolically engineered Lactococcus lactis by increasing ATP demand. Appl Microbiol Biotechnol 100:9509-9517.

44. Casadaban MJ, Cohen SN. 1980. Analysis of gene control signals by DNA fusion and cloning in Escherichia coli (MC1000). J Mol Biol 138:179-207.

45. Solem C, Dehli T, Jensen PR. 2013. Rewiring lactococcus lactis for ethanol production. Appl Environ Microbiol 79:2512-2518.

46. Maguin E, Prévost H, Ehrlich SD, Gruss A. 1996. Efficient insertional mutagenesis in lactococci and other gram-positive bacteria. J Bacteriol 178:931-935.

Tables and Figures

Table 1. Strains and plasmids used in this work

\begin{tabular}{|c|c|c|}
\hline Strain and plasmid & Relevant genotype & Source or reference \\
\hline \multicolumn{3}{|l|}{ Strains } \\
\hline MG1363 & Wild-type L. lactis subsp. & (19) \\
\hline & cremoris & \\
\hline MG363-M & MG1363 adapted on mannitol & $(16)$ \\
\hline Ace001 & MG1363 $\Delta^{3} l d h, \Delta p t a, \Delta a d h E$ & $(43)$ \\
\hline
\end{tabular}


$\triangle b u t B A$

Ace001-M

Ace001 adapted on mannitol

AceF

Ace001-M $\Delta m t l F$ (previously

addressed AceM)

Ace R

Ace001-M $\Delta m t l R$

This work

Ace001C

Ace001 $\triangle c c p A$

This work

AceCF

Ace001-M $\triangle m t l F, \Delta c c p A$

This work

AceCR

Ace001-M $\triangle m t l R, \Delta c c p A$

This work

Ace001-mtlR

mtlR overexpressed Ace001,

This work

20\% TPI promoter

Ace001-mtlD

mtlD overexpressed Ace001,

This work

20\% TPI promoter

MC1000

E.coli cloning host

DH10B

high-efficiency competent $E$.

coli host

Plasmids

pTD6

pTD6 A derivative of pAK80

containing a gusA

reporter gene

pPmtla $^{\text {WT }}$

pTD6 with wild type $m t l A$

This work

promoter preceding gusA

reporter gene

pPmtla $^{\text {mut }}$

pTD6 with C-39T mutant $m t l A \quad$ This work

promoter preceding gusA

reporter gene

pPmtlA $^{\text {mutII }}$

pTD6 with G-46T mutant mtlA This work

promoter preceding gusA 
reporter gene

pPmtlD

pTD6 with $m t l D$ promoter

This work

preceding gusA reporter gene

pPmtlAD

pTD6 with fused $m t l A$ and

$m t l D$ promoter preceding gusA

reporter gene

pJET1.2

high-efficiency cloning

ThermoFisher Scientific

plasmid for blunt-ended

ligation

pG' ${ }^{+}$host8

E. coli/L. lactis shuttle vector,

$\operatorname{Tet}^{\mathrm{R}}$, thermosensitive replicon

pKmtlR

Used for knocking out $m t l R$

This work

pKCepA

Used for knocking out $c c p A$

This work

Table 2. Effect of mutations in the mannitol operon promoter on expression level

\begin{tabular}{ccccc}
\hline Sugars & Host & $\mathrm{P}_{\mathrm{mtlA}, \mathrm{WT}}$ & $\mathrm{P}_{\mathrm{mtlA}, \mathrm{C}-39 \mathrm{~T}}$ & $\mathrm{P}_{\mathrm{mtlA}, \mathrm{G}-46 \mathrm{~T}}$ \\
\hline Glucose & MG1363 & $0.01 \pm 0.01$ & $15.6 \pm 0.9$ & $0.43 \pm 0.01$ \\
Glucose & MG1363M & $0.06 \pm 0.01$ & $52.16 \pm 2.0$ & $1.17 \pm 0.11$ \\
Mannitol & MG1363M & $449 \pm 5$ & $4415 \pm 56$ & $4183 \pm 34$
\end{tabular}

590 Notes: $\mathrm{P}_{\text {mtlA,WT}}$, wild type $m t l A$ promoter; $\mathrm{P}_{\mathrm{mtlA}, \mathrm{C}-39 \mathrm{~T}}$, $m t l A$ promoter containing the C-39T

591 mutation; $\mathrm{P}_{\mathrm{mtlA}, \mathrm{G}-46 \mathrm{~T}}$, mtlA promoter containing the G-46T mutation. Data were collected at 592 exponential phase and the promoter activities were shown as Miller Units. All treatments were duplicated and deviations was indicated. 
Table 3. Overview of mtlA and mtlD promoter strength in different regulatory conditions

\begin{tabular}{|c|c|c|c|c|}
\hline \multirow{2}{*}{ Sugar } & \multirow{2}{*}{ Strains } & \multirow{2}{*}{ Promoters } & \multicolumn{2}{|c|}{ Miller units } \\
\hline & & & Exponential & Stationary \\
\hline Glucose & Ace001 & $m t l D$ & $0.00 \pm 0.00$ & $0.00 \pm 0.00$ \\
\hline Glucose & AceR & $m t l D$ & $0.01 \pm 0.01$ & $0.01 \pm 0.00$ \\
\hline Glucose & Ace $001 \mathrm{M}$ & $m t l D$ & $2.38 \pm 0.10$ & $122 \pm 5$ \\
\hline Mannitol & Ace $001 \mathrm{M}$ & $m t l D$ & $871 \pm 46$ & $1699 \pm 137$ \\
\hline Glucose & Ace001 & $m t l A^{\mathrm{WT}}$ & $0.03 \pm 0.00$ & $0.05 \pm 0.01$ \\
\hline Glucose & Ace001 & $m t l A^{\mathrm{Mut}}$ & $15.6 \pm 1.1$ & $40.0 \pm 0.4$ \\
\hline Glucose & AceR & $m t l A^{\text {Mut }}$ & $14.5 \pm 0.9$ & $41 \pm 2$ \\
\hline Glucose & Ace $001 \mathrm{M}$ & $m t l A^{\mathrm{Mut}}$ & $44.3 \pm 2.2$ & $2273 \pm 164$ \\
\hline Mannitol & Ace $001 \mathrm{M}$ & $m t l A^{\mathrm{Mut}}$ & $4078 \pm 230$ & $7539 \pm 687$ \\
\hline Glucose & Ace001C & $m t l A^{\mathrm{WT}}$ & $588 \pm 15.7$ & $1880 \pm 86$ \\
\hline Glucose & Ace001C & $m t l A^{\mathrm{Mut}}$ & $523 \pm 13.1$ & $1842 \pm 48$ \\
\hline Glucose & Ace001C & $m t l D$ & $405 \pm 6.6$ & $894 \pm 12$ \\
\hline Glucose & AceCF & $m t l A^{\mathrm{WT}}$ & $3787 \pm 232$ & $6055 \pm 351$ \\
\hline Glucose & AceCF & $m t l A^{\mathrm{Mut}}$ & $3413 \pm 70$ & $6375 \pm 308$ \\
\hline Glucose & AceCF & $m t l D$ & $1955 \pm 65$ & $3664 \pm 88$ \\
\hline Glucose & AceCR & $m t l A^{\text {Mut }}$ & $39.0 \pm 3.5$ & $52.2 \pm 1.5$ \\
\hline Glucose & AceCR & $m t l D$ & $0.00 \pm 0.00$ & $0.06 \pm 0.01$ \\
\hline
\end{tabular}




\begin{tabular}{cccc}
\hline Strain & Genotype & Growth on mannitol & Mannitol production \\
\hline Ace001 & MG1363 $\Delta l d h, \triangle a d h E, \Delta p t a, \Delta b u t B A$ & - & - \\
Ace001M & Ace001 adapted on mannitol & + & + \\
AceF & Ace001M $\Delta m t l F$ & - & + \\
Ace001-mtlR ${ }^{\uparrow}$ & $m t l R$ overexpressed Ace001 & - & + \\
AceR & Ace001M $\Delta m t l R$ & - & - \\
Ace001C & Ace001 $\Delta c c p A$ & + & + \\
AceCF & AceF $\Delta c c p A$ & - & + \\
AceCR & AceR $\Delta c c p A$ & - & -
\end{tabular}

600

601

602

603

604

605

606

Table 5. The effects of deleting CcpA on mannitol production

\begin{tabular}{ccccc}
\hline Condition & Strains & $\begin{array}{c}\text { Mannitol produced } \\
(\mathrm{g} / \mathrm{L})\end{array}$ & $\begin{array}{c}\text { Yield } \\
(\%)\end{array}$ & ${\text { Biomass }\left(\mathrm{OD}_{600}\right)}$ \\
\hline $\mathrm{TSF}$ & AceF & 6.08 & 0.6 & - \\
$\mathrm{TSF}$ & AceCF & 5.92 & 0.59 & - \\
$\mathrm{TSF}$ & AceR-mtlD & & - \\
$\mathrm{AG}$ & AceF & 10.1 & 0.55 & 1.38 \\
$\mathrm{AG}$ & AceCF & 2.18 & 0.41 & 0.65 \\
$\mathrm{AG}$ & AceR-mtlD & & 0.34 & 0.47 \\
& & 0.62 & 0.34 & \\
\hline
\end{tabular}


607 TSF, two-step fermentation; AG, Anaerobic growth, $35 \mathrm{mM}$ arginine were added; the 608 biomass and yield were measured and calculated after 7 days incubation.
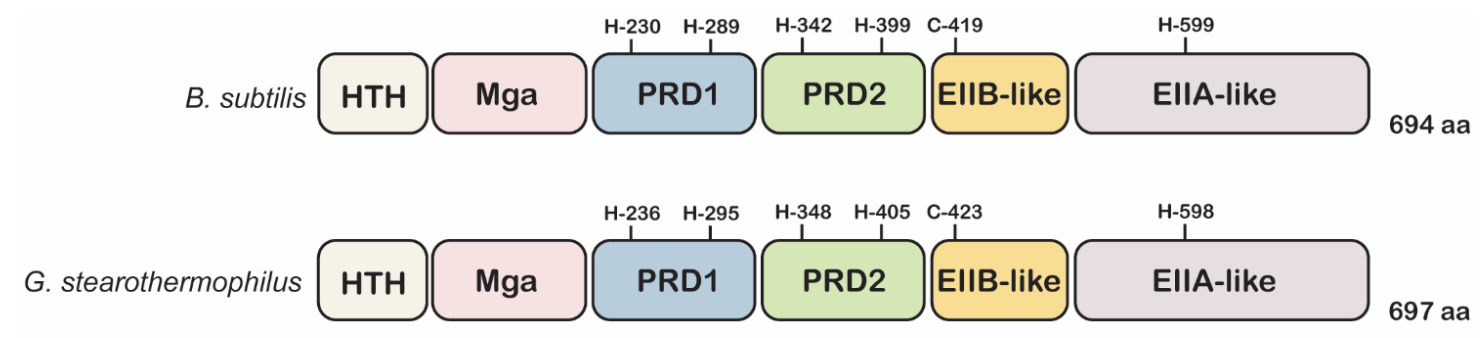

609

610

Figure 1. Protein structure of the characterized mtlR from $B$. subtilis, $G$.

611 stearothermophilus and L. casei. The phosphorylation sites were indicated with the amino

612 acid and their position. 
a
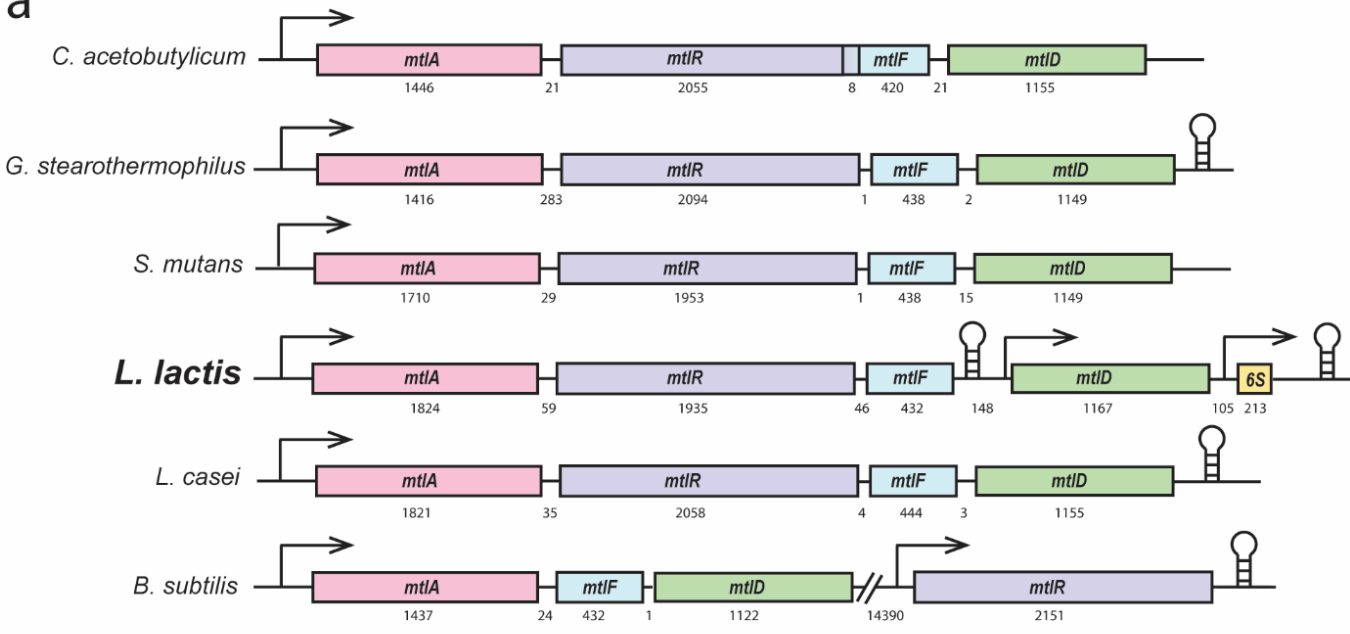

b

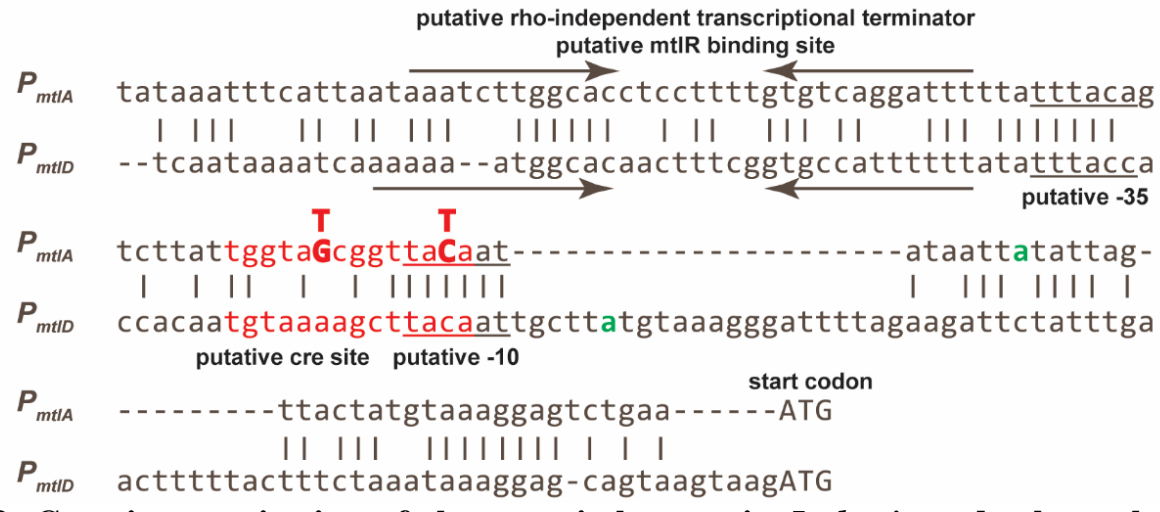

Figure 2. Genetic organization of the mannitol genes in L. lactis and other selected

firmicutes, as well as putative promoters upstream mtlA \& mtlD in L. lactis. Arrows

represent characterized or predicted promoters. The numbers beneath the genes in a) indicate either gene length or length of the spacers between indicated genes. 6S, 6S RNA. In b), putative transcriptional elements are indicated. The transcriptional start sites are indicated with green color; cre sites are indicated with red color, the inverse repeat sequence, i.e., the $m t l R$ binding site was indicated with arrows; -10 and -35 elements are underscored. The mutations C-39T and G-46T (the numbers indicate the distance from start codon ATG) in mtlA promoter region gained during mannitol adaptation are indicated with a red capital bold letter. 

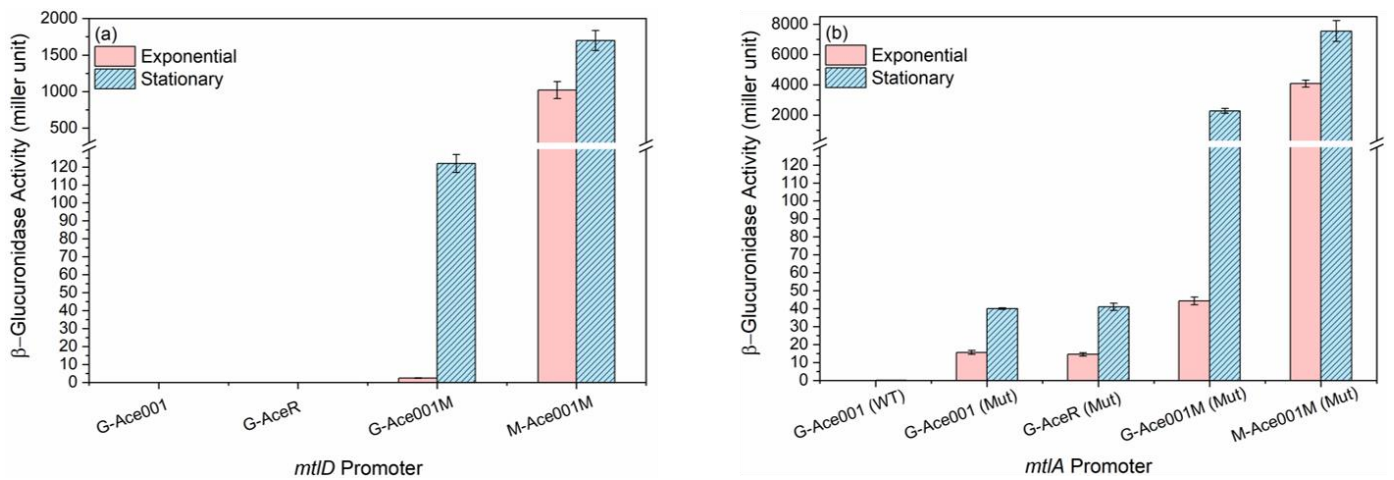

Figure 3. Testing the effect of $\boldsymbol{m t l R}$ on expression of mannitol genes. (a) The effect on

$626 m t l D$ promoter expression. (b) The effect on $m t l A$ promoter expression, WT, wild type $m t l A$

627 promoter; Mut, mtlA promoter with C-39T mutation. The carbon source is indicated with G

628 (1\% glucose) or M (1\% mannitol). The host is indicated with Ace001, AceR, Ace001M. Cells

629 harvested in exponential phase are indicated with red color, while cells harvested in

630 stationary phase are indicated with blue color. Experiments were carried out in duplicates,

631 and standard deviations are indicated with error bars. 


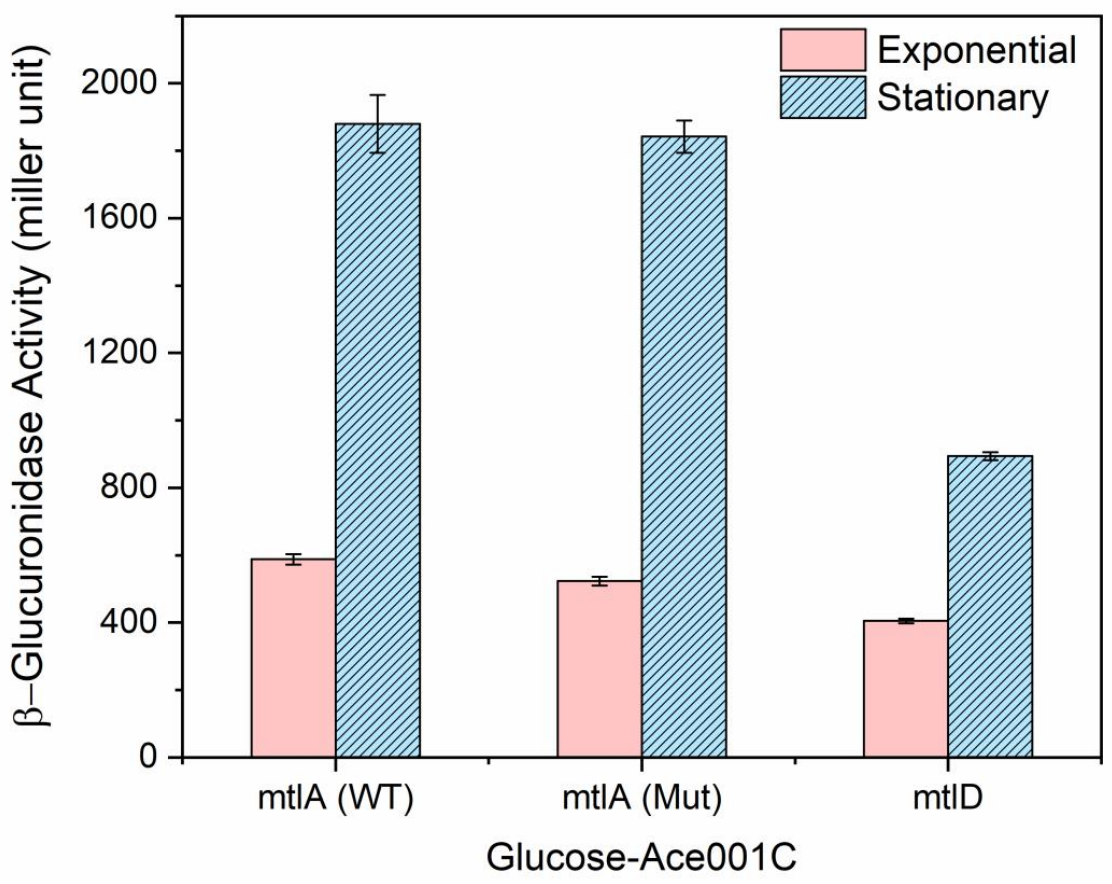

632

633 Figure 4. The activity of $m t l A$ and $m t l D$ promoters in Ace001C. WT, wild type $m t l A$ 634 promoter; Mut, mtlA promoter with $\mathrm{C}-39 \mathrm{~T}$ mutation. Cells are grown in $1 \%$ glucose and 635 harvested at exponential phase (red color), stationary phase (blue color). Experiments were 636 carried out in duplicates and standard deviations indicated by error bars. 


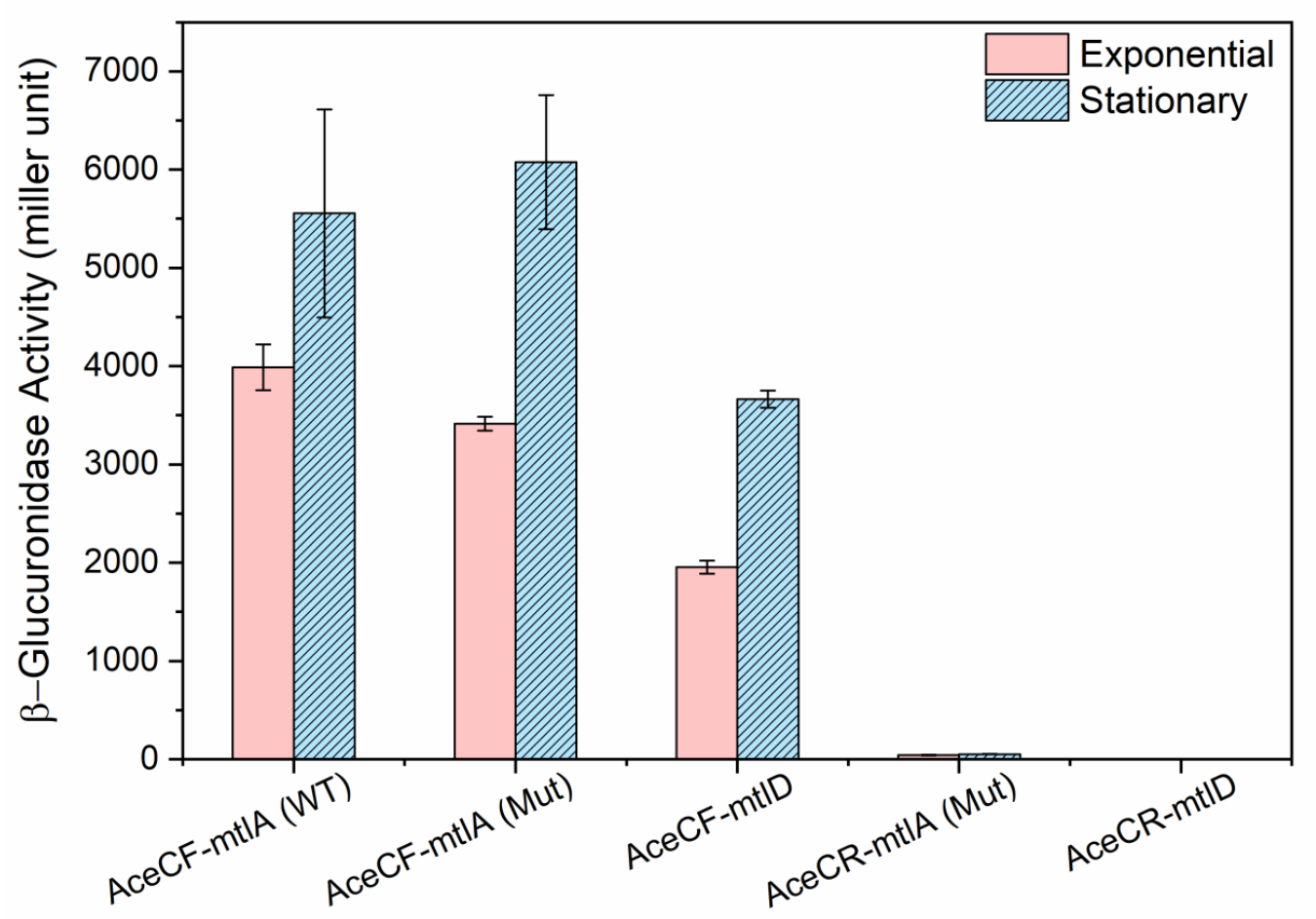

\section{Glucose}

Figure 5. The activity of $m t l A$ and $m t l D$ promoters in AceCF and AceCR. WT, wild type mtlA promoter; Mut, $m t l A$ promoter with C-39T mutation. Cells were growing in $1 \%$ glucose and harvested at exponential phase (red color), stationary phase (blue color). Experiments were carried out in duplicates and standard deviations are indicated with error bars.

Glucose

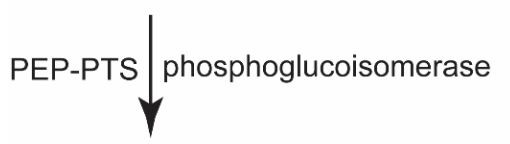

F6P
Mannitol

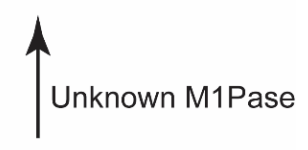

M1P

Figure 6. Scheme of mannitol production in L. lactis. PEP-PTS, phosphoenolpyruvate (PEP)-dependent glucose phosphotransferase system (PTS); F6P, fructose-6-phosphate; M1PDH, mannitol-1-phosphate 5-dehydrogenase; M1P, Mannitol-1-phosphate. 


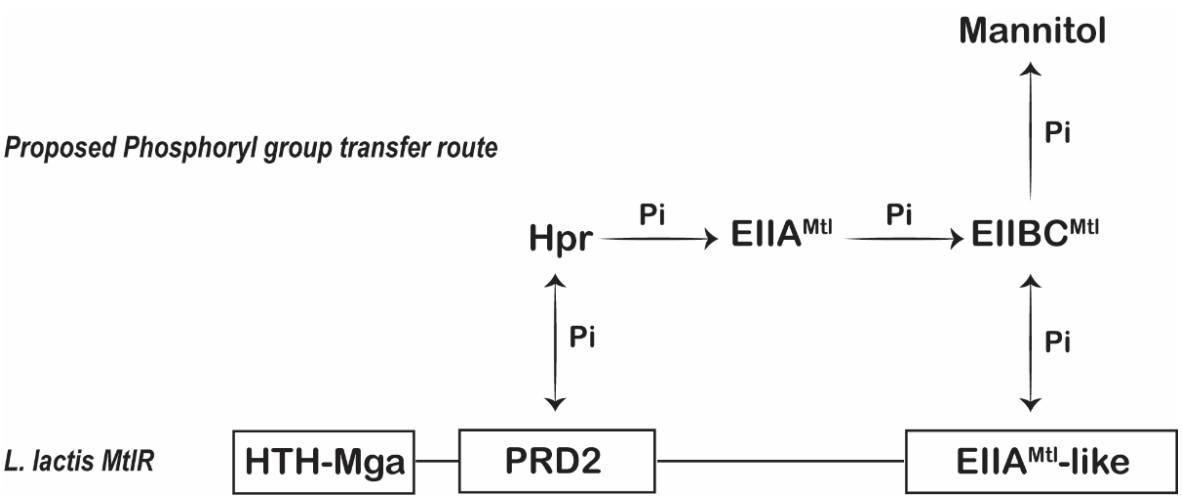

647 Figure 7. The proposed phosphoryl group transfer route in L. lactis MtIR. 
a

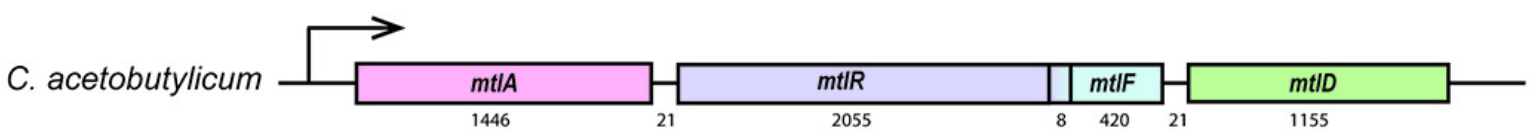

G. stearothermophilus

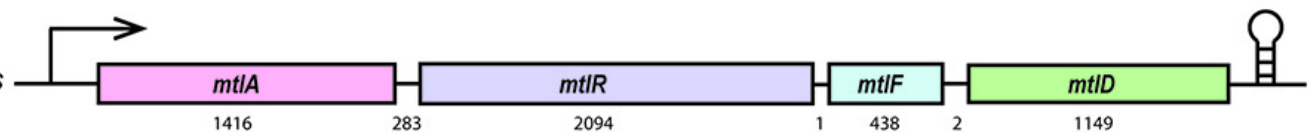

S. mutans

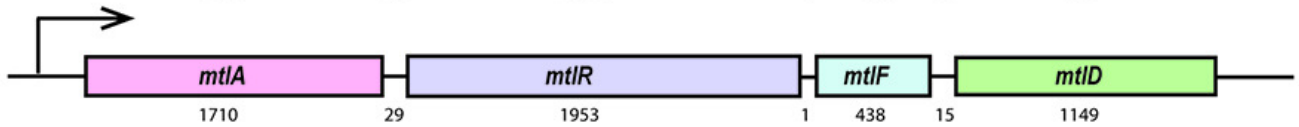

L. lactis
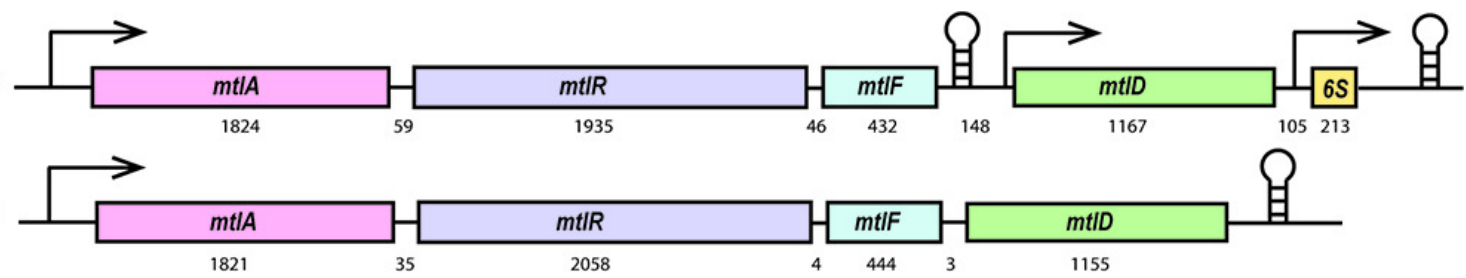

$\mathrm{b}$

putative rho-independent transcriptional terminator putative $\mathrm{mtl}$ R binding site

$\boldsymbol{P}_{\text {mt/A }}$ tataaattcattaataatcttggcacctcctttgtgtcaggattt

I III II II III IIIII I II III II III IIIIII

$\boldsymbol{P}_{\text {mtID }}$--tcaataaaatcaaaaaa--atggcacaactttcggtgccattttttatatttacca $\mathbf{T} \quad \mathbf{T}$ putative $-\mathbf{3 5}$

$\boldsymbol{P}_{\text {mt/A }}$ tcttattggtaGcggttaCaat--.-.-.-.-.-.--.---ataattatattag-

$\boldsymbol{P}_{\text {mtID }}$ ccacaatgtaaaagcttacaattgcttatgtaaagggattttagaagattctatttga putative cre site putative -10

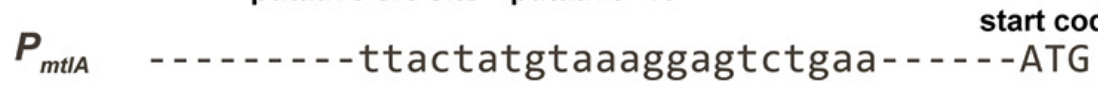

$\boldsymbol{P}_{\text {mtID }}$ actttttactttctaaataaaggag-cagtaagtaagATG 

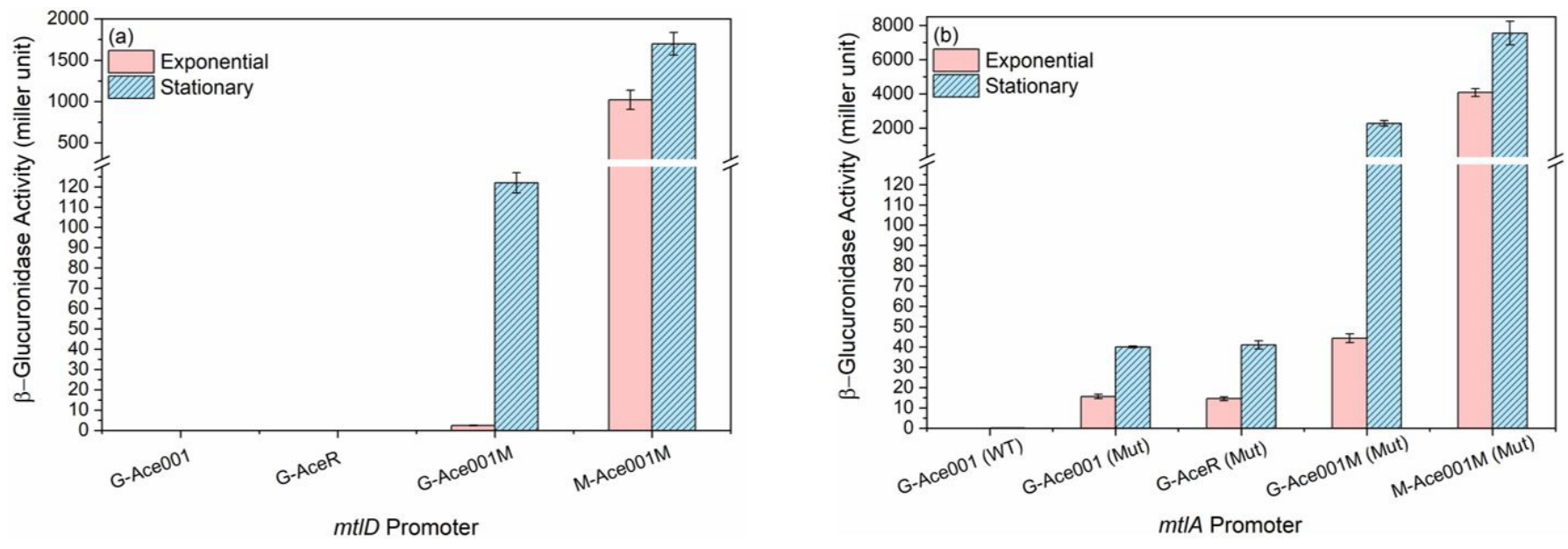


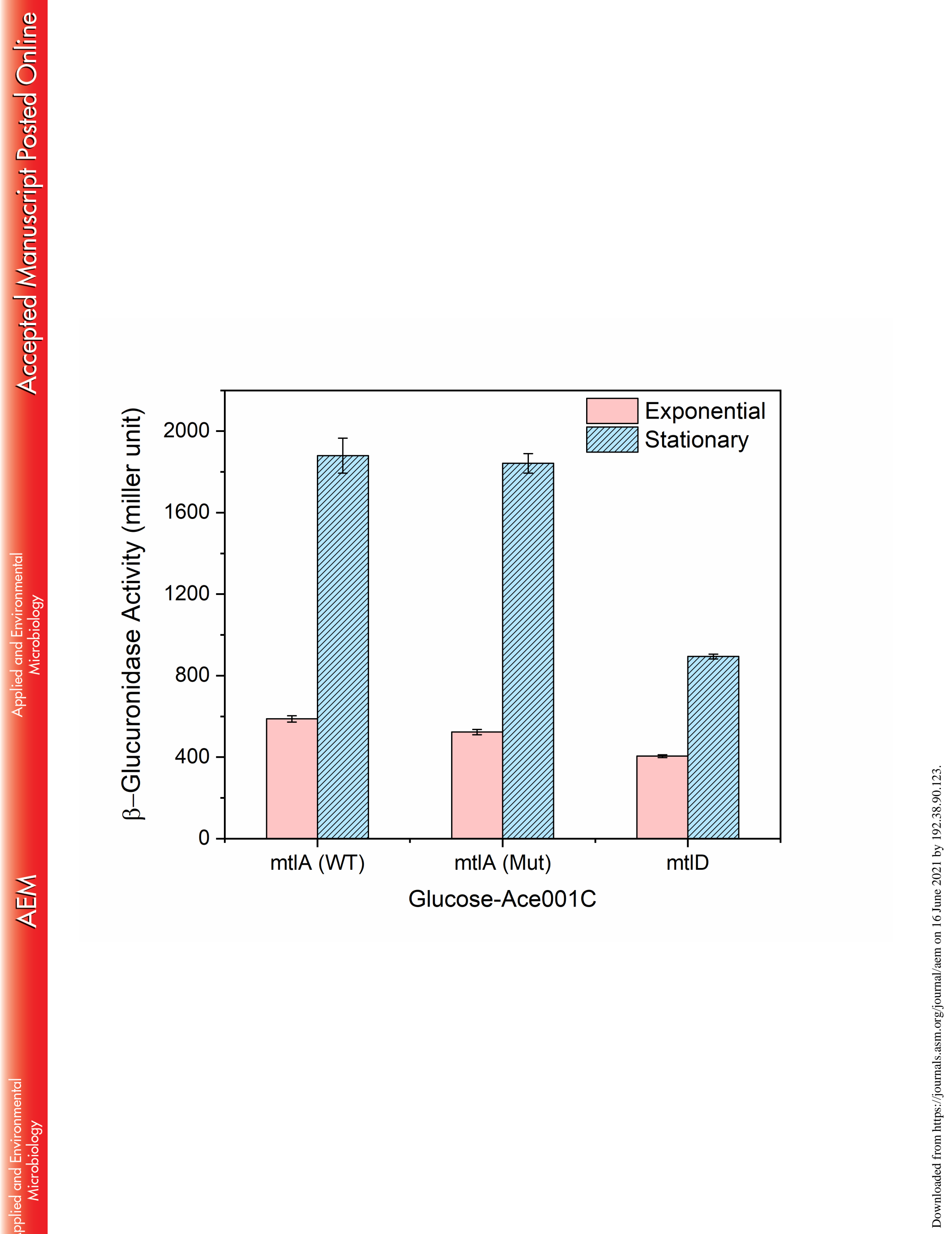




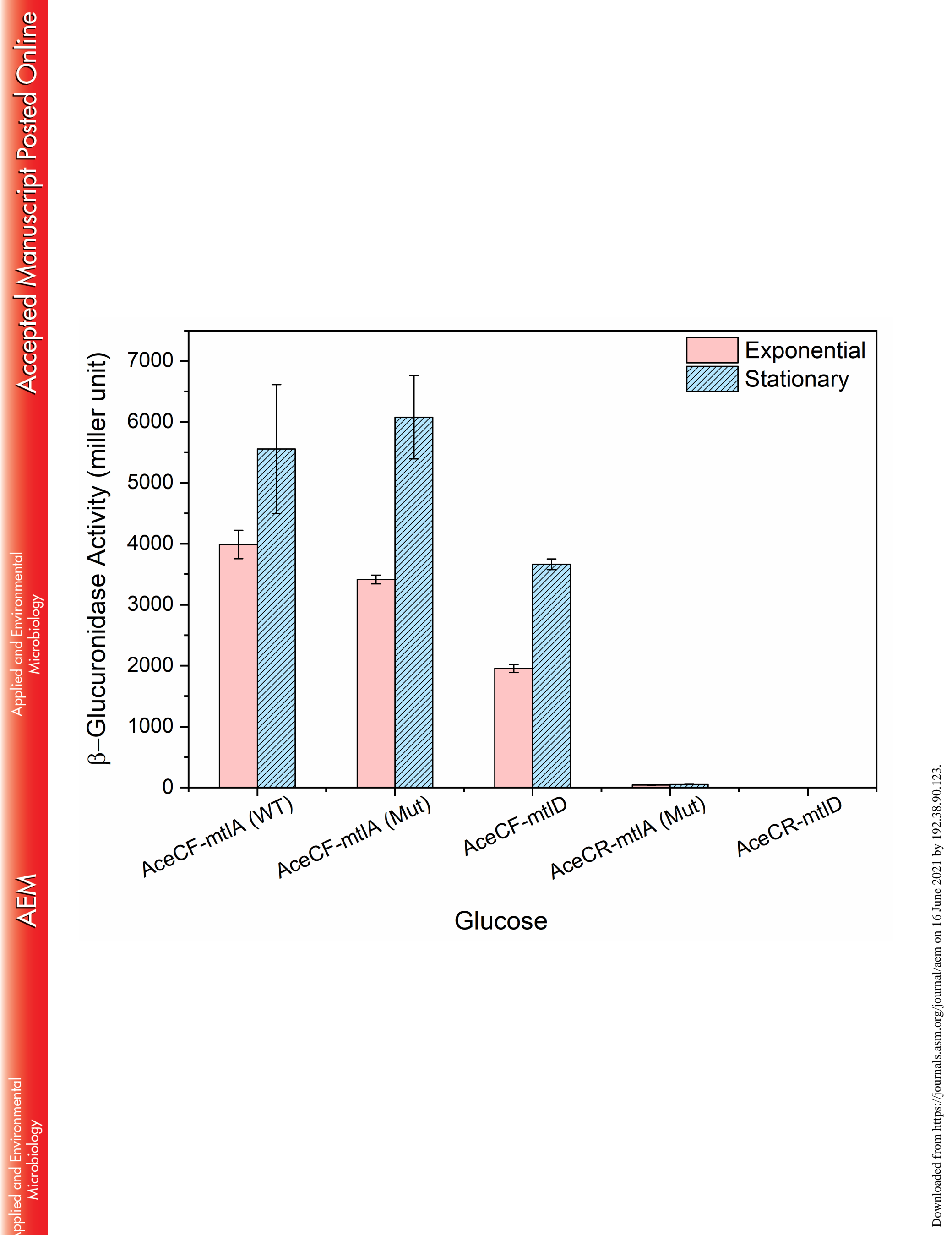


